\title{
Agôn
}

Revue des arts de la scène

$5 \mid 2012$

L'entrée en scène

\section{Les parades du Radeau}

Le dispositif d'entrées dans le travail de François Tanguy

\section{Maxence Cambron}

\section{(2) OpenEdition}

Journals

Édition électronique

URL : http://journals.openedition.org/agon/2424

DOI : 10.4000/agon.2424

ISSN : 1961-8581

Éditeur

Association Agôn

Référence électronique

Maxence Cambron, «Les parades du Radeau », Agôn [En ligne], 5 | 2012, mis en ligne le 25 janvier 2013, consulté le 01 mai 2019. URL : http://journals.openedition.org/agon/2424; DOI : 10.4000/ agon. 2424

Ce document a été généré automatiquement le 1 mai 2019.

Association Agôn et les auteurs des articles 


\title{
Les parades du Radeau
}

\author{
Le dispositif d'entrées dans le travail de François Tanguy
}

\section{Maxence Cambron}

1 Pénétrer sur la piste d'un cirque, cet « 0 » de sciure (pour paraphraser Shakespeare) bordé par la foule émotive, c'est franchir le seuil d'un univers où bêtes et hommes cohabitent sous les accents musicaux des cuivres et des percussions. Au sens propre du mot comme dans le jargon technique, pour l'artiste, apparaître sur la piste, c'est faire son «entrée ». Il y a au cirque une certaine mythologie de l'entrée dont l'antécédence immémoriale remonte à l'Antiquité, lorsque les combattants apparaissaient dans l'arène bondée, sous les hourras de spectateurs enivrés par l'odeur du sang. Le cirque moderne n'est certes plus le spectacle sanguinaire des anciens Romains, mais il conserve néanmoins cette force d'engouement générée par l'atmosphère de risque et de danger permanent auxquels s'exposent les artistes. L'entrée du dompteur, du trapéziste, du cracheur de feu, comme l'entrée des héros; héros mythologiques (re)jouant devant nous les luttes fondatrices de l'humanité.

2 Il y a donc dans le cirque une part de poésie et de métaphore qui s'adresse sans intermédiaire à la mémoire collective. Sur ou au-dessus de la piste, cercle primitif, représentation de l'infini, du tout contenu et de la rotondité de la Terre, le dompteur, l'acrobate ou le jongleur figurent l'Homme universel ; non pas issus d'une mythologie particulière qui imposerait son cadre narratif déterminé, mais d'une structure de croyance générale commune à toutes les civilisations, invoquée pour éclaircir l'inexplicable (la création du monde, le rythme des saisons, la mort...). Le dompteur, c'est l'Homme aux prises avec les fauves dans la nature hostile, mais aussi l'homme qui apprivoise la bête pour l'asservir; le jongleur, l'Homme aux prises avec les éléments (le feu par exemple) qui, comme son collègue, dompte la flamme et la vainc; en haut, sur son fil de fer, le funambule est l'Homme métaphysique, en équilibre précaire, qui traverse les airs, côtoyant la chute, côtoyant la mort. Tout le chapiteau se métamorphose alors en vaste représentation mentale, suscitant les passions de spectateurs en empathie face à la fiction de ces hommes et ces femmes assaillis par les risques; une exploration des passions humaines, aux confins de leur purgation, qui fait dialoguer le cirque et le théâtre 1 . 
3 En contrepoint de cette atmosphère morbide, l'entrée de clown apparaît comme une respiration venue pour relâcher les crispations. Dénuée de statut «officiel », comme le précise Agnès Pierron, «l'entrée de clown [...] n'est pas au programme. Ce n'est qu'un petit en-cas pour la route ${ }^{2}$ ", une transition entre deux numéros, une façon de " remplir » le temps de latence imposé par la manutention de la piste. Cette position intermédiaire est à la fois une cause et une conséquence de la nature de l'art du clown. Sa vocation, en effet, est de tourner en dérision le sérieux, d'exagérer les traits de la bêtise humaine et, particulièrement au cirque, de déjouer l'angoisse entre deux instants de forte tension. Entre les cris et les peurs, les rires provoqués par les entrées de clowns font planer sur l'ensemble de la représentation une ambiance de vanité où le grotesque cohabite avec le sublime, l'ombre de la mort avec la complexité de la vie; une vanité qui s'étend à l'ensemble des numéros, qui se révèlent tous comme des représentations d'une part métaphysique de l'Homme. Souvent teintées de violence (chutes, bagarres, cascades improbables...), les entrées de clowns sont des condensés de nature humaine, sans limite physique et sans borne au ridicule. Malgré le contraste qu'elles sont censées apporter, elles se rangent sans peine au même niveau que tous les autres numéros de la revue métaphysique.

\section{De l'entrée et de l'avant-garde}

4 Ce n'est que peu de temps après leur apparition en Angleterre dans le courant du XIX siècle, que le cirque et le music-hall (un art frère, sorte de développement du cirque dans une salle de théâtre, avec moins d'animaux mais plus de danseuses...) attirèrent les regards des artistes, de tous les horizons. Dans les trente dernières années du XIXe siècle en effet, on sent poindre une forme de fascination pour les saltimbanques ainsi qu'un véritable attrait esthétique pour les lieux, les costumes, les couleurs dans lesquels ils évoluent, bien loin des codes académiques alors en vigueur. Les peintres parisiens, fréquentant le Cirque d'Hiver ou le Cirque Fernando (futur Medrano) furent les premiers à consacrer des pans de leur œuvre à cet univers artistique nouveau³. De même, poètes et romanciers installèrent leurs rêveries et leurs personnages autour des pistes et sur les strapontins ${ }^{4}$. Nombreux sont les artistes (sans oublier les musiciens) pour qui le cirque fut donc une passion, une inspiration.

5 Arts populaires par excellence, les arts forains furent assimilés aux « résidus d'un âge fabuleux ${ }^{5}$ ", un Âge d'Or pourtant emprunt d'une modernité toute actuelle. Le monde nouveau du XIXe siècle, celui de la révolution industrielle, était en passe de devenir le monde de la vitesse, de l'instantanéité et de la succession mécanique. Les revues de cirque, comme celle de music-hall, suivirent ce mouvement en composant des spectacles d'une suite d'entrées sans narration, détachées les unes des autres, dans un rapport d'immédiateté complète avec l'instant. À la recherche de formes nouvelles, les artistes virent dans le cirque le renouveau formel de l'art dans le décloisonnement hiérarchique entre les Beaux-Arts et les autres, et surtout dans la modernité.

6 Les avant-gardes des débuts du XX siècle poursuivirent le geste amorcé en l'ouvrant aux arts vivants. Danse et théâtre furent alors le terrain d'expérimentations privilégié de formes et de techniques d'expression nouvelles, leur permettant (d'un art vivant à un autre) de sortir de la sclérosante rigueur académique, pour faire trembler les traditions d'un souffle de légèreté et de liberté. Fascinés par les formes, les couleurs, la créativité indépendante d'impératifs formels, la virtuosité physique et, pour ainsi dire, le « devenir- 
animal» des artistes, la musicalité permanente et, comme nous le suggérions précédemment, la pureté immédiate des émotions générées, des chorégraphes et des metteurs en scènes puisèrent dans les arts forains le ferment d'un renouveau esthétique majeur'. En point d'orgue, la bouffonnerie foraine, centre de la vanité circassienne, cristallise l'attrait des avant-gardes pour ces arts simples et humbles, alimentant généreusement le mythe déjà ancien de l'artiste maudit et solitaire, errant sur les routes de ville en ville tel un vagabond ou un exilé.

7 C'est d'ailleurs à partir de ce dernier point qu'à la génération suivant ces premières avant-gardes, Kantor formera l'axe fondamental de son œuvre. À la tête de sa compagnie, le Théâtre Cricot 2 (dont le nom même signifie « cirque » en polonais), il prit de lui-même la position de l'artiste forain. Comme il le confie dans l'un de ses derniers essais, «La Baraque foraine ", écrit en amont de Wielopole-Wielopole (1982) :

Bien que, au cours des différentes périodes qui se sont succédées, aux différentes « étapes » et « arrêts » de mon chemin j'aie écrit sur des pierres milliaires les noms des endroits: Théâtre Informel, Théâtre Zéro, Théâtre Impossible, Théâtre de la Réalité dégradée, Théâtre Voyage, Théâtre de la Mort, quelque part à l'arrière-plan il y avait toujours cette même BARAQUE DE FOIRE. [...]

Après tant de conflits et beaucoup d'étapes franchies, aujourd'hui je vois assez clairement le chemin déjà parcouru et je comprends pourquoi j’ai toujours refusé avec tant d'obstination un statut officiel et institutionnel. Ou plutôt : pourquoi ont été refusés avec tant d'obstination à moi et à mon théâtre les privilèges et les conditions découlant de ces positions sociales. Parce que mon théâtre a toujours été une Baraque de foire. Le vrai Théâtre de l'Émotion.'

8 On saisit ici la fascination fondamentale de Kantor pour les forains. Déconsidérés, chassés, mais indispensables dans une société du loisir, ils sont mis dans une position d'illégalité ambivalente : refusant d'emblée ce que de toute façon on ne leur autoriserait pas. $\mathrm{Ce}$ motif de l'illégalité ou de la clandestinité, omniprésent dans l'œuvre du créateur de Qu'ils crèvent les artistes, traduit donc la référence au cirque en position politique, s'affirmant, depuis la marge, comme un contestataire transgressif, bouleversant et caricaturant les codes d'une société cadenassée.

Il ne fait donc pas de doute que le recours à la liberté formelle du cirque et du music-hall, où joyeusetés côtoient les pires frayeurs au rythme effréné de la fanfare, n'est pas apparu tout à fait par hasard à la charnière des deux derniers siècles précédents : derrière l'espoir du retour à l'Âge d'or suscité par la marche folle du progrès, se cachait déjà la sourde inquiétude des crises à venir. La foire, son tumulte, sa beauté convulsive et son agressivité latente (empreinte d'animalité fauve et de violence morbide) ne pouvait qu'être choisie par les artistes impatients mais inquiets du renouveau. Si, un siècle plus tard, il semble que foire, cirque et music-hall aient perdu de leur influence, peut-être même relégués à l'arrière-plan des spectacles surannés, ce n'est que parce qu'ils ont disparu des discours d'artistes, supplantés, par un phénomène analogue à leur propre promotion, par les nouvelles technologies et leur dramatisation possible. Or, à l'époque de la transdisciplinarité et de l'acteur polyvalent (acteur mais aussi danseur, musicien, chanteur, régisseur...), l'héritage de la foire ne semble guère épuisé tant il continue d'irriguer (discrètement) nos regards contemporains. C'est pourquoi nous nous proposons d'analyser le travail d'un artiste français actuel (dont on percevra le portrait en creux dans les premiers propos de cet article) chez qui œuvre ce principe de transdisciplinarité, mais où la filiation avec les arts forains est encore bien présente, tout 
comme celle qui le lie à Kantor, et particulièrement dans le dispositif des entrées et des numéros, qui soutient l'ensemble de son processus de création.

\section{Le « cirque allégorique » de François Tanguy}

Depuis 1982, François Tanguy œuvre au sein du Théâtre du Radeau où il met à jour patiemment, à raison (et en moyenne) d'une création tous les trois ans, un continuum théâtral unique peuplé de fantômes ; fantômes de la peinture, du cinéma, de la littérature, de la philosophie et du théâtre, au premier rang desquels prend place Kantor (une rencontre artistique majeure et marquante dont on perçoit l'empreinte dans le travail du metteur en scène de la compagnie mancelle dès ses premières créations). La forte influence de Kantor dans les années 1980 et 1990 a pu se mesurer selon différents critères avec plus ou moins de pertinence, principalement tangible par les dehors esthétiques des spectacles concernés. Chez François Tanguy, l'apport esthétique de Kantor est certes fondamental mais il accompagne surtout une communion de vues sur l'art du théâtre, la place de l'artiste dans la société et le regard sur l'Histoire. Voilà pourquoi la notion de baraque foraine, que nous évoquions plus haut, prend chez François Tanguy une importance et des résonances toutes kantoriennes.

11 Transitant donc par la figure de Kantor, cette affirmation de l'art pauvre du cirque et des forains en tant que pratique assumée du théâtre, constelle toute la geste artistique de François Tanguy au Théâtre du Radeau. Nous en voulons pour preuve les éléments précoces mais fondateurs qui, dans les trois créations de la seconde moitié des années 1980 (années de la «révélation » du Théâtre du Radeau, notamment par le Festival d'Automne), marquèrent cette forte présence. Verbalement d'abord dans le titre de la création de 1989, Woyzeck - Büchner - Fragments forains, où il ne fait aucun doute que c'est sous ce signe que fut interprété le texte de Büchner, et dans Jeu de Faust (1987), spectacle divisé en multiples séquences, dont une nommée "Cirque allégorique ». En 1986 enfin, Mystère bouffe mettait en perspective le théâtre médiéval de la place publique et l'opérabouffe, bouffon, clownesque. Sur scène, l'environnement esthétique ne trahit pas cette intuition. Un matériau y est omniprésent : le bois (bois brut, bois pauvre, bois trouvé). Des tables, des chaises, des bancs mais surtout des planches, qui servent à la construction de vastes boîtes dans lesquelles évoluent les acteurs - aménagement indispensable de la baraque foraine traditionnelle. Les acteurs, qui s'assimilent à des artistes de cirque, comme Laurence Chable, interprétant dans Jeu de Faust une clownesse sortant d'une petite boîte en bois montée sur roulettes, poussée par une sorte de dompteur affublé d'un hautde-forme et d'un fouet (Jean Rochereau). Après six années d'absence, elle revint au cœur de deux entrées majeures, l'une dans Choral (1994) et l'autre dans Bataille du Tagliamento (1996). L'autre évocation du cirque s'est faite beaucoup plus directement par l'accueil, au sein de la distribution, d'un duo d'artistes issus du cirque, Branlo \& Nigloo, jouant les acrobates dans Choral. Notons enfin les liens très intimes que le Théâtre du Radeau entretient avec les compagnies itinérantes comme le Théâtre Dromesko, avec qui ils formèrent Le Campement, expérience artistique commune (qui visita Strasbourg, Venise, Rennes, Nancy, Prague, Weimar...) au moment de l'acquisition par le Théâtre du Radeau de la fameuse Tente, chapiteau moderne accueillant depuis 1997 leurs répétitions et certaines représentations.

12 À travers ces quelques exemples, on saisit la forte présence du cirque dans les origines créatrices de François Tanguy. Au regard des créations récentes (Ricercar (2007) ou 
Onzième (2011), il peut apparaître cependant que cette influence se soit estompée. Or il faudrait constater plutôt une mutation de ces premières sources d'inspiration en de nouvelles formes d'expression du langage théâtral, directement liées à cet héritage forain. Au cœur de cette mutation, on trouve le dispositif des entrées, dont le principe premier est à peine transformé. Les spectacles du Radeau, comme une parade de cirque ou de music-hall, se constituent d'un enchaînement de séquences autonomes mais reliées les unes aux autres selon une exigence d'invisibilité des «coutures» qui les relient. Cet enchaînement d'entrées, dans le double sens du mot, irrigue l'organisation du plateau sur trois aspects fondamentaux : l'espace, la dramaturgie et le jeu de l'acteur.

\section{L'espace des entrées}

13 L'espace des spectacles du Théâtre du Radeau est un espace complexe, composite, lieu d'expression des formes, des perspectives mais surtout des matières. Le bois dominant des premières créations a progressivement cédé sa place à une grande variété de matériaux : les tissus, le papier (papier-peint), les matières plastiques et surtout le métal, pour lui-même et pour son utilité dans la construction de cadres, de châssis, de tables, qui font évidemment dialoguer l'espace théâtral du Radeau avec la piste du cirque moderne, aménagée d'agrès principalement métalliques. Et tout comme s'il s'agissait d'agrès réels, les acteurs s'accrochent aux châssis pour diverses acrobaties, à l'instar de Laurence Chable faisant, dans Onzième, son entrée accrochée à un cadre métallique perché sur deux tiges poussé par une autre comédienne (une entrée qui fait référence à celle de la clownesse du Jeu de Faust). Tout en prononçant le texte de Kafka sur la danseuse Eduardowa tiré des premières pages du Journal, Laurence Chable amorce, dans cette position contorsionnée et inconfortable, quelques mouvements d'une danse étrange, ayant surtout pour but de lui faire varier sa position. Dans Ricercar, une vieille porte sert de planche à acrobatie où tous les hommes de la distribution grimpent un à un avant de se laisser glisser au sol. Numéros de danse et de voltige grotesques.

14 La scène encombrée de ces dizaines de toiles, d'écrans, de cadres, de chaises et de tables ne partage en rien le minimalisme du cercle vide de la piste de cirque. Il semble en revanche que nous soyons les témoins du curieux ballet des agrès dans la remise aux décors, là où stationnent les artistes avant leur entrée en piste, accompagnés de leurs supports métalliques. La scène du Radeau revêt ainsi l'apparence d'un lieu de transition où la succession des entrées en scène pourrait aussi bien être celle des sorties de pistes. Les acteurs et les actrices évoluent dans un tournoiement d'agrès, précédant, suivant ou accompagnant leurs apparitions. À l'ouverture de chaque nouvelle séquence, à l'approche de chaque entrée, c'est un mouvement quasi général du plateau qui met en branle les éléments, agençant ainsi l'espace pour de nouveaux développements, comme la piste s'aménage pour permettre au nouveau numéro de débuter.

\section{Une dramaturgie de l'intermittence}

15 Comme nous le suggérions dans la première partie de notre propos, l'un des principaux attraits du cirque, pour les artistes de l'avant-garde des premières années du XXe siècle, repose sur l'absence totale de contrainte formelle imposant au créateur la fabrication d'un récit selon un certain nombre de normes traditionnelles destinées au façonnage d'une intrigue architecturée, de telle manière que le lecteur, ou le spectateur, puisse en 
retracer toute l'évolution, de l'exposition au dénouement. La succession des entrées dans une revue de cirque traditionnel ne correspond en effet à aucune volonté de narration, même infime. Il ne s'agit que d'une suite de numéros agencés selon des critères à la fois techniques (concernant la gestion des agrès par exemple) et spectaculaires (parcours et progression des émotions des spectateurs, le numéro "clou» pour finir etc.). Une importante partie de la pratique et de la théorie artistiques du XXe siècle s'est inspirée de cette liberté pour revendiquer une souplesse formelle créatrice. Ainsi Artaud affirmant dès 1931 dans "La mise en scène et la métaphysique » un abandon du texte et de ses corollaires narratifs sous peine de créer un «théatre d'idiot, de fou, d'inverti, de grammairien, d'épicier, d'anti-poète et de positiviste, c'est-à-dire d'Occidental ${ }^{8}$ » ou Deleuze et Guattari ayant recours au rhizome pour définir ces œuvres où « n'importe quel point [...] peut être connecté avec n'importe quel autre ${ }^{9}$ » sans qu'il soit jamais question de continuité logique arborescente. Nous n'extrayons pas par hasard du long chaînon d'artistes et de penseurs du postdramatique ${ }^{10}$ ces trois noms, car ils forment un socle de pensée fondamental pour François Tanguy qui vécut, dans ses années de formation intellectuelle et de premières expériences artistiques, l'explosion novatrice de la pensée deleuzienne.

L'influence croisée de ces trois sources dans le travail de François Tanguy est manifeste : l'élaboration dramaturgique en est une preuve patente. Constitués par l'assemblage de séquences dramatiques (composées aussi bien par l'agencement de l'espace, par le texte, le son et la mise en lumière), les spectacles du Théâtre du Radeau ne suivent en aucun cas le développement d'une intrigue. Ce qui rassemble ces séquences et les relie en une chaîne cohérente (dont une part importante du travail consiste à en effacer les coutures) sont avant tout des critères formels d'occupation de l'espace, de progression visuelle et de distribution (le contenu et le sens des textes est certes compris dans ces critères, mais pas dans un sens narratif). On retrouve donc ici les bases de l'entrée et de la revue, auxquelles s'ajoute l'esthétique de la fragmentation, directement inspirée de Kafka (dont les fragments narratifs servent d'origine à un très grand nombre de situations dramatiques des entrées du Radeau).

Ce principe de séquences fragmentaires, indépendantes mais (selon le motif rhizomatique) connectables les unes aux autres, se pose donc comme une entrave à toute narration. Pour conserver le vocabulaire deleuzien, on définira même ces entrées comme des milieux, n'ayant donc ni origine ni conclusion, comme l'est ce brin d'herbe décrit par Kafka, et cité par les auteurs de Mille plateaux, qui « ne commence à croître qu'au milieu de la tige ${ }^{11} »$. On rejoint une fois encore l'entrée des saltimbanques, et particulièrement celle des clowns telle que nous rappelions sa fonction (définie par Agnès Pierron) « de petit en-cas pour la route», d'élément transitoire entre deux numéros «officiels", inscrits dans le programme. En la rapportant aux entrées du Radeau, cette idée met en perspective la fonction dramaturgique des séquences avec l'apparence du plateau telle que nous la décrivions précédemment : si la scène est une coulisse, alors ce qui s'y déroule est à côté de l'événement, un commentaire au passage, un instant provisoire et décentré. Cette impression n'aura jamais été aussi forte que dans Coda (2004) où de nombreux textes étaient dédiés à la description d'événements non représentés en scène. Traversant l'espace dans toutes les configurations, les acteurs semblent être les manutentionnaires d'un monde hors-champ, condamnés qu'ils sont à le décrire par bribes, comme des restes mémoriels d'un espace-temps fantomatique. 


\section{L'impossible entrée du personnage}

18 Ces intermittences de l'œil ne peuvent reposer que sur un mouvement alternatif. Aux entrées (littérales cette fois) succèdent nécessairement des sorties. Cette binarité classique du théâtre prend, dans les spectacles du Théâtre du Radeau, une dimension chorégraphique destinée à amplifier ou, au contraire, à banaliser l'apparition/disparition des acteurs, par des entrées spectaculaires ou des sorties forcées. Toutes les modalités de surgissement ou d'évanouissement mettent donc en avant la présence de l'acteur au cœur même du dispositif d'entrées. Car c'est l'acteur qui fait l'entrée et aucune autre instance fictive.

19 L'entrée concrète est un seuil ; au seuil d'une entrée de music-hall ou de cirque, pas plus qu'il n'y a de narration, il n'y a de personnage (exception faite du clown). Au Théâtre du Radeau, le personnage empêché répond à la narration entravée: conséquence du processus de fractionnement des éléments de la représentation où la présence de l'acteur, devenant instantanée et inaliénable à la progression d'un être fictif, génère des entités passagères comme autant de diffractions d'un être kaléidoscopique. C'est la raison pour laquelle on ne pourra suivre une entité identifiée (principalement et presque exclusivement par son costume) d'un bout à l'autre d'une représentation: son existence étant limitée à la durée d'une séquence où elle apparaît. Chaque entrée est donc un recommencement, une re-naissance, une apparition dont l'aboutissement (au bout de quelques minutes, voire quelques secondes) demande à l'acteur la force de concentration et d'imagination de ne faire apparaître qu'une évocation, qu'une silhouette, à l'opposé de toute composition sur la durée d'un spectacle.

Le dispositif des entrées au Théâtre du Radeau s'inscrit donc dans une histoire théatrale (et picturale) forte, où la narration, et le sens, sont relégués au profit de la fragmentation et de l'hétérogénéité. Dans une suite éclatée d'entrées circassiennes, la seule histoire possible serait donc celle du corps de l'acteur, traversant, coupant, arpentant l'espace scénique dans une série de séquences cloisonnées. Entités floues aux existences conditionnées par des bornes serrées, évoluant dans un espace encombré de cadres, de châssis et de tables prêts à supporter leurs acrobaties fantoches, ces silhouettes rejoignent les artistes du cirque par leur allure mythologique. Ni personnages ni être réels, leurs actions les assimilent aux allégories peuplant le fonds commun de nos représentations mentales, nos archétypes ancestraux. Ces parades allégoriques transforment la scène en lanterne magique vivante où, à chaque apparition, comme sur l'enluminure ou la carte de tarot, c'est le fantôme d'une mémoire qui entre, livrée aux spectateurs sous la forme d'une énigme, non à intellectualiser mais à ressentir par toutes les pores des émotions : ne pas comprendre, mais perdre connaissance au seuil de la piste du cirque allégorique. 


\section{BIBLIOGRAPHIE}

ARTAUD, Antonin. Le Théâtre et son double [1938]. Paris : Gallimard / Folio essais, 1985.

DELEUZE, Gilles, GUATTARI, Félix. Capitalisme et schizophrénie 2 : Mille plateaux. Paris : Editions de Minuit, 1980.

GENET, Jean. Le Funambule [1958]. Paris : Gallimard / Folio, 2009

KANTOR, Tadeusz. Le Théâtre de la Mort [1977]. Lausanne, L'Âge d'homme, 2004.

LEHMANN, Hans-Thies. Le Théâtre postdramatique [1999]. Paris, L'Arche, 2002, trad. P.-H. Ledru.

MANGANARO, Jean-Paul. François Tanguy et le Radeau. Paris, P.O.L., 2007.

PIERRON, Agnès. Dictionnaire de la langue du cirque, Des mots dans la sciure. Paris, Stock, 2003.

TACKELS, Bruno. François Tanguy et le Théâtre du Radeau. Besançon, Les Solitaires intempestifs / Ecrivains de plateau, 2005.

\section{NOTES}

1. Il reste cependant que le cirque se distingue par son immédiateté ainsi que par le refus ou l'empêchement de toute "intellectualité ». Si au théâtre la notion de risque n'est jamais tout à fait absente, au cirque, le spectateur est indéniablement plongé dans un état de peur et de tension. Cette mise à l'épreuve nerveuse du spectateur fait toujours de l'homme (l'artiste) le sujet de la peur : peur de la chute du funambule, peur de l'imprévisibilité des fauves, peur de l'erreur, peur de la mort. À ces peurs successives répondent des réactions immédiates de l'ordre du réflexe et de la pulsion : cris, sursauts, mains devant les yeux. Jouant avec nos peurs profondes, le cirque est, de fait, le domaine des émotions pures (entendons instinctives), le lieu de l'affect, tandis que le théâtre joue sur l'intellect et les émotions médiatisées.

2. Agnès, Pierron, Dictionnaire de la langue du cirque, Des mots dans la sciure, Paris, Stock, 2003, pp.258-259.

3. Renoir peignant en 1868 un clown violoniste seul sur la piste et plus tard deux jeunes jongleuses ; Degas, saisissant au vol Miss Lola, la voltigeuse du cirque Fernando, en 1879 ; Seurat (La Parade du cirque en 1888 et son écuyère en 1891) ou encore Toulouse-Lautrec et ses nombreux croquis d'artistes en plein numéro.

4. Théophile Gautier, spectateur assidu et passionné du cirque, fit des fauves un motif de sa Comédie de la mort, comme Baudelaire, autre passionné, qui y puisa ses questionnements sur le rire et l'effroi.

5. Jean, Genet, Le Funambule [1958], Paris, Gallimard, coll. Folio, 2009.

6. Le double exemple de Schlemmer et Meyerhold est certainement le plus représentatif de cette période d'intense renouvellement artistique. Le Ballet triadique de Schlemmer (1922), chorégraphie emblématique du Bauhaus, est à n'en pas douter la réinterprétation formelle et radicale des couleurs, des formes et des agissements des clowns, des jongleurs et des acrobates (esthétique qui annonce celle $d u$ "nouveau cirque ", notre contemporain.). Il en est de même pour Meyerhold, chez qui l'expérimentation théâtrale d'une haute exigence physique (la biomécanique) et tout l'agencement de la scène, entre futurisme et constructivisme, reflète l'engouement secret et non conventionnel du metteur en scène russe pour l'art du clown, du 
mime et du théâtre forain, acquis dans ses années de formation (aux alentours de 1910) et traduit dans son œuvre dans les années 1930.

7. Tadeusz, Kantor, «La Baraque foraine » in Denis, Bablet (dir.) Le Théâtre de la Mort [1977], Lausanne, L'Âge d'homme, 2004, p. 272.

8. Antonin, Artaud, «La mise en scène et la métaphysique » in Le Théâtre et son double [1938], Paris, Gallimard, coll. Folio Essais, 1985, p. 61.

9. Gilles, Deleuze, Félix, Guattari, « Rhizome » in Capitalisme et schizophrénie 2: Mille plateaux, Paris, Editions de Minuit, 1980, p. 13.

10. Hans-Thies, Lehmann, Le Théâtre postdramatique [1999], Paris, L'Arche, 2002, trad. P.-H. Ledru. 11. Franz, Kafka, Journal, Paris, Grasset, 1973, trad. M. Robert cité par Gilles, Deleuze, Félix, Guattari, Capitalisme et schizophrénie 2 : Mille plateaux, op. cit., p. 34.

\section{RÉSUMÉS}

Cet article prend pour point de départ le vocabulaire du cirque où l'« entrée » désigne d'abord le numéro de clown dans son entier et, par extension, tous les numéros. À la charnière des XIX et $\mathrm{XX}^{\mathrm{e}}$ siècles, les entrées de cirque (alors en pleine apogée), ont cristallisé un grand nombre de revendications novatrices et libératrices des avant-gardes naissantes. Sur les traces de ces avantgardes, le théâtre a été le plus profondément reconsidéré à la lumière des nouvelles exigences de l'art moderne : instantanéité, absence de narration, fragmentation, énergie et violence, pureté des émotions.

En nous appuyant sur le travail de François Tanguy, metteur en scène du Théâtre du Radeau depuis 1982, nous analyserons les survivances formelles des entrées de cirque dans le théâtre contemporain suivant le sillon kantorien de la « baraque foraine ».

\section{INDEX}

Mots-clés : cirque, avant-garde, Tanguy (François), Kantor (Tadeusz)

\section{AUTEUR}

\section{MAXENCE CAMBRON}

Maxence Cambron est doctorant (CEAC) et chargé de cours en arts de la scène à l'université Charles-de-Gaulle - Lille 3. Sa thèse, dirigée par Claude Jamain, intitulée « Le Théâtre de la remembrance : moyens et enjeux d'une représentation de la mémoire en scène », s'attache à décrypter ce qui, dans le théâtre contemporain principalement, met en œuvre un sentiment de mémoire dans la production artistique, et ses conséquences sur la réception du spectacle vivant. Par ailleurs comédien et metteur en scène, il dirige depuis 2005 sa propre compagnie : Les Arpenteurs. Cette saison, il met en scène Collection pour deux voix, spectacle adapté des Diablogues de Roland Dubillard. maxence.cambron[at]orange.fr 